\title{
Actitud de los docentes frente a los estudiantes con necesidades educativas especiales, Amazonas, Perú, 2020
}

\section{Attitude of teachers towards students with special educational needs, Amazonas, Peru, 2020}

\author{
Charito de Jesús Távara Sabalú1, Flor Teresa García Huamán²
}

\section{RESUMEN}

El objetivo de la presente investigación fue determinar la actitud de los docentes frente a los estudiantes con necesidades educativas especiales. El estudio fue descriptivo, de corte transversal. La población estuvo constituído por 184 docentes de la Universidad Nacional Toribio Rodríguez de Mendoza de Amazonas, Chachapoyas, región Amazonas. La muestra estuvo representada por 106 docentes. El muestreo fue probabilístico completamente al azar. Se encontró que el 100\% de los encuestados no cumplen con los indicadores de la dimensión cognitiva, es decir no tienen grado académico en educación inclusiva tampoco especialización, diplomados, cursos o capacitaciones en educación inclusiva. Todos los docentes encuestados cumplen con la dimensión afectiva, cumpliendo con los indicadores apoyo académico de acuerdo a la necesidad especial, 98.1\%; proporciona tiempo adicional a la clase, 97.2\% y motiva antes de iniciar la clase el 99.1\%. El 100\% de los encuestados cumplen con los indicadores de la dimensión conductual, el $94.3 \%$ prepara o prepararía material educativo adicional, el 100\% adapta o adaptaría la sesión de aprendizaje, el 93.4\% aplica o aplicaría una evaluación diferenciada, el 99.1\% acompaña o acompañaría académicamente a sus estudiantes. Se concluye que la actitud de los docentes frente a los estudiantes con necesidades educativas especiales es positiva por que cumplen con las dimensiones: afectiva y conductual.

Palabras clave: Estudiantes especiales, necesidades educativas.

\begin{abstract}
The objective of the present research was to determine the attitude of teachers towards students with special educational needs. The study was descriptive, cross-sectional. The population consisted of 184 teachers from the National University Toribio Rodríguez de Mendoza de Amazonas, Chachapoyas, Amazonas region. The sample was represented by 106 teachers. The sampling was probabilistic completely at random. It was found that $100 \%$ of the respondents do not meet the indicators of the cognitive dimension, that is, they do not have an academic degree in inclusive education, nor do they have specialization, diplomas, courses or training in inclusive education. All the teachers surveyed comply with the affective dimension, fulfilling the academic support indicators according to special need, 98.1\%; provides additional time to the class, $97.2 \%$ and motivates before starting the class $99.1 \%$. $100 \%$ of the respondents meet the behavioral dimension indicators, $94.3 \%$ prepare or prepare additional educational material, 100\% adapt or adapt the learning session, $93.4 \%$ apply or apply a differentiated evaluation, $99.1 \%$ accompany or he would accompany his students academically. It is concluded that the attitude of teachers towards students with special educational needs is positive because they meet the dimensions: affective and behavioral.
\end{abstract}

Keywords: Special students, educational needs..

${ }^{1}$ Docente nombrada de la Institución Educativa $N^{\circ}$ 1005, Jorge Chávez Dartnell. Licenciada en educación primaria, Maestría en orientación y tutoría.Correo Electrónico: charito_tavara@hotmail.com

${ }^{2}$ Docente Principal de la Facultad de Ingeniería y Ciencias Agrarias de la Universidad Nacional Toribio Rodríguez de Mendoza de Amazonas. Biólogo-Microbiólogo, Magister en Ciencias con Mención en Gestión Ambiental, Doctora en Ciencias Ambientales y Especialista en Gestión de la Biodiversidad..Correo Electrónico: flor.garcia@untrm.edu.pe 


\section{INTRODUCCIÓN}

La actitud es un factor que determina diferentes situaciones y lleva al individuo a tomar decisiones o reaccionar a cierta situación, lo conduce actuar de una forma concreta y específica y normalmente esta forma es constante (Stahlberg y Frey 1991, citados en Castillo, 2007).

Baron y Byrne (2002), ven la actitud como reacción afectiva positiva $o$ negativa hacia un objeto o proposición abstracta o concreta y señalan que las actitudes son aprendidas, tienden a permanecer bastante estables con el tiempo, están dirigidas siempre hacia un objeto o idea particular y poseen los componentes cognoscitivo, afectivo y conductual.

El desarrollo de actitudes favorables hacia las diferencias individuales del alumnado es un tema difícil de abordar por la complejidad de factores que intervienen en ellas (Sanhueza, et al., 2012). Un alto porcentaje de docentes atribuye tanto el éxito como el fracaso de los logros de los estudiantes a la capacidad del alumno y a su situación familiar (Aguado, et al., 2008). Más allá de la inversión en recursos o la transformación de las estructuras, los procesos innovadores exitosos son aquellos fundamentados en el cambio de los docentes (Essomba, 2006)

Las actitudes de los docentes hacia los alumnos con necesidades educativas especiales (NEE) pueden verse influenciadas por varios factores como la responsabilidad del docente que forma al estudiante, la posición de los educadores frente al rendimiento académico de sus alumnos con NEE; las consideraciones de los docentes respecto a su propia formación y conocimientos, así como los recursos con que cuentan para atender a los alumnos con NEE; la opinión de los docentes en cuanto a la disciplina y el orden en el aula de clase compartida por estudiantes con NEE y alumnos regulares; los beneficios percibidos por los docentes respecto a la socialización de los alumnos con NEE gracias a la integración en el aula; desarrollo emocional referido a la posición del docente frente a la integración en el aula como elemento que contribuye o no en el desarrollo emocional de los estudiantes con NEE, entendiendo como el proceso de formación de su identidad, autoestima y confianza en sí mismos y las creencias, referido a la opinión de los docentes frente a la integración en el aula como una práctica educativa deseable. (Mula et al., 2002).

Uno de los aspectos más valorados para llevar a cabo la tarea de atender alumnos con necesidades NEE es el trabajo colaborativo, este aspecto ya ha sido documentado por Zerpa (2002), Diaz y Hernández
(2002) y Teran y Pachano (2009), quienes concluyeron que los alumnos que trabajan cooperativamente aprenden más, desarrollan una actitud más positiva, establecen mejores relaciones con sus compañeros, aumentan su autoestima y aprenden tanto valores como habilidades sociales en forma más afectiva.

En Chile la educación especial ha experimentado avances importantes en términos de incremento de la subvención en esta modalidad educativa y la generación de un cuerpo normativo que ha permitido la integración escolar de los estudiantes con discapacidad al sistema de educación regular (Ministerio de Educación chile, 2005).

En Costa Rica (2008) se ratificó la Convención de los Derechos Humanos de las personas con discapacidad y su protocolo facultativo, de la organización para las Naciones Unidas, que exige a los estados que garanticen un sistema de educación inclusiva en todos los niveles. La política educativa reflejada en las directrices técnico administrativas del Ministerio de Educación Pública, relativas a los servicios de apoyo para estudiantes con NEE que asisten a la educación regular, pone de manifiesto un esfuerzo formal para favorecer la educación inclusiva (Sanhueza, et al., 2012).

La Declaración Universal de los Derechos Humanos de 1948, explicita el derecho fundamental a la educación; el informe Warnock, 1978, precisa el concepto de educación especial no centrada en las deficiencias que presentan las personas sino en las necesidades educativas que poseen, entendiendo la educación especial como un continuo de prestación de apoyos temporales o permanente desde el currículum común; el foro consultivo internacional sobre educación para todos enfatiza el acceso a la educación y la equidad para el apendizaje; la Declaración de Salamanca y marco de acción señala las condiciones necesarias para la integración de los estudiantes con necesidades educativas especiales al sistema común (Echeita y Verdugo, 2004).

Es necesario mencionar que en el Perú, la Ley 29973 , Ley General de la Persona Con Discapacidad establece en su artículo 37, la calidad del servicio educativo, las instituciones educativas de las diferentes etapas, modalidades y niveles del sistema educativo nacional están obligadas a realizar las adaptaciones metodológicas y curriculares, así como los ajustes razonables necesarios para garantizar el acceso y permanencia del estudiante con discapacidad y el artículo 39 se refiere a la formación superior en discapacidad, las universidades, institutos y escuelas superiores, públicos y privados, incluyen asignaturas sobre discapacidad en los 
currículos y programas para la formación de técnicos y profesionales en los campos de la educación, el derecho, la medicina, la sicología, la administración, la arquitectura, la ingeniería, la economía, la contabilidad y el trabajo social.

Por lo anteriormente mencionado el objetivo de la presente investigación fue determinar la actitud de los docentes frente a los estudiantes con necesidades educativas especiales.

\section{MATERIAL Y MÉTODO}

El estudio fue descriptivo, de corte transversal. La población estuvo constituído por 184 docentes (nombrados:113 y contratados:71) de la Universidad Nacional Toribio Rodríguez de Mendoza de Amazonas, región Amazonas. La muestra estuvo representada por 106 docentes. El muestreo fue probabilístico completamente al azar, en las nueve facultades que oferta la universidad estudiada.

Tabla1. Porcentaje de docentes de nueve facultades de la Universidad Nacional Toribio Rodríguez de Mendoza que representaron la muestra de estudio.

\begin{tabular}{l|ccc}
\hline \multicolumn{1}{c}{ Facultades muestre adas } & Frecuencia (número de docentes) & Porcentaje (\%) \\
\hline 1 & Facultad de Ciencias Económicas y administrativas & 4 & $3.8 \%$ \\
\hline 2 & Facultad de Ingeniería Civil y Ambiental & 16 & $15.1 \%$ \\
\hline 3 & Facultad de Ingeniería y Ciencias Agrarias & 16 & $15.1 \%$ \\
\hline 4 & Facultad de Zootecnia Biotecnología y Agronegocios & 0 & $0.0 \%$ \\
\hline 5 & Facultad de Educación y Ciencias de la Comunicación & 14 & $13.0 \%$ \\
\hline 6 & Facultad de Ciencias Sociales & 10 & $9.4 \%$ \\
\hline 7 & Facultad de Derecho y Ciencias Políticas & 13 & $12.3 \%$ \\
\hline 8 & Facultad de Ciencias de la Salud & 16 & $15.1 \%$ \\
\hline 9 & Facultad de Ingeniería de Sistemas y Mecánica Eléctrica & 17 & $16.0 \%$ \\
\hline & & 106 & $100.0 \%$ \\
\hline
\end{tabular}

Se utilizó como instrumento de recolección de datos una encuesta, la misma que fue validada a través de juicio de expertos, así mismo se aplicó la prueba de confiabilidad de Kuder Richardson, obteniéndose un coeficiente: $\operatorname{Kr}(20)=0.76$. La aplicación de la encuesta se realizó previo consentimiento informado, de manera virtual y anónima.

La encuesta comprendió 12 preguntas para determinar la actitud de los docentes frente a los estudiantes con necesidades educativas especiales. Se evaluaron tres dimensiones: Dimensión cognitiva a través de su formación académica; dimensión afectiva de acuerdo a su sensibilidad y dimensión conductual en relación a las necesidades de aprendizaje de sus estudiantes.

Los indicadores evaluados fueron en la dimensión cognitiva: Grado académico, especialización, diplomados, cursos, capacitaciones en TICs para educación inclusiva; en la dimensión afectiva: Apoyo académico, proporciona tiempo adicional, motivación antes de iniciar la clase y en la dimensión conductual: Prepara o prepararía material educativo adicional, adapta o adaptaría la sesión de aprendizaje, aplica o aplicaría una evaluación diferenciada, acompaña o acompañaría académicamente a sus estudiantes.

\section{RESULTADOS}

Tabla 2. Porcentaje de cumplimiento de indicadores de la dimensión cognitiva.

\begin{tabular}{|c|c|c|c|c|}
\hline \multirow[b]{2}{*}{$\mathbf{N}^{\circ}$} & \multirow[b]{2}{*}{ Indicadores de dimensión cognitiva } & \multicolumn{2}{|c|}{ Cumplimiento del indicador } & \multirow[b]{2}{*}{ TOTAL (\%) } \\
\hline & & SI (\%) & NO $(\%)$ & \\
\hline 1 & Grado académico & 0 & 100 & 100 \\
\hline 2 & Especialización en educación inchusiva & 0 & 100 & 100 \\
\hline 3 & Diplomado en educación inclusiva & 0 & 100 & 100 \\
\hline 4 & Cursos en educación inclusiva & 0 & 100 & 100 \\
\hline 5 & Capacitación en TICs para educación inclusiva & 0 & 100 & 100 \\
\hline
\end{tabular}

Tabla 3. Porcentaie de cumplimiento de indicadores de la dimensión afectiva.

\begin{tabular}{|c|c|c|c|c|}
\hline \multirow[b]{2}{*}{$\mathbf{N}^{\circ}$} & \multirow{2}{*}{ Indicadores de dimensión afectiva } & \multicolumn{2}{|c|}{ Cumplimiento del indicador } & \multirow[b]{2}{*}{ TOTAL (\%) } \\
\hline & & SI (\%) & NO $(\%)$ & \\
\hline 1 & A poyo a ca démico de acuerdo a la necesidad especial & 98.1 & 1.9 & 100 \\
\hline 2 & Proporciona tiempo adicional a la clase & 97.2 & 2.8 & 100 \\
\hline 3 & Motivación antes de iniciar la clase & 99.1 & 0.9 & 100 \\
\hline
\end{tabular}


Tabla 4. Porcentaje de cumplimiento de indicadores de la dimensión conductual.

\begin{tabular}{|c|c|c|c|c|}
\hline \multirow[b]{2}{*}{$\mathbf{N}^{\circ}$} & \multirow[b]{2}{*}{ Indicadores de dimensión conductual } & \multicolumn{2}{|c|}{ Cumplimiento del indicador } & \multirow[b]{2}{*}{ TOTAL (\%) } \\
\hline & & SI $(\%)$ & NO $(\%)$ & \\
\hline 1 & Prepara o prepararía material educativo adicional & 94.3 & 5.7 & 100 \\
\hline 2 & Adapta o adaptaría la sesión de aprendizaje & 100 & 0 & 100 \\
\hline 3 & Aplica o aplicaría una evaluación diferenciada & 93.4 & 6.6 & 100 \\
\hline 4 & Acompaña o acompañaría academicamente a sus estudiantes & 99.1 & 0.9 & 100 \\
\hline
\end{tabular}

\section{DISCUSIÓN}

La educación especial ha ido experimentando una serie de cambios, influida por declaraciones, informe, foros, conferencias internacionales e investigaciones que han aportado con fuerza la idea del derecho a contar con una educación de calidad para todos y todas. (Sanhueza, et al., 2012).

El docente juega un papel muy importante en la educación inclusiva $\mathrm{y}$, por tanto, su actitud es un eslabón primordial para el éxito. Al respecto, durante los últimos cuarenta años se han llevado a cabo numerosas investigaciones acerca de las características de edad, clase social, experiencia, salario, entrenamiento recibido y actitud que deben tener los docentes eficientes e incluyentes (Gonzáles y Triana, 2018).

Una de las premisas más importantes para la consecución de la inclusión educativa es el cambio en la forma de enseñanza, que conlleva la capacitación de los docentes para que aprendan a trabajar con personas que se salen de lo preestablecido (Gonzáles y Triana, 2018), en esta investigación se encontró que el $100 \%$ de los encuestados no están capacitados, no cumplen con los indicadores de la dimensión cognitiva, es decir no tienen grado académico en educación inclusiva tampoco especialización, diplomados, cursos o capacitaciones en educación inclusiva (Tabla 2), estos datos concuerdan con los estudios de Sanhueza, et al. (2012) en la necesidad de capacitar a los docentes $(71 \%)$. De igual manera pareciera ser que los profesores de Chile (43\%) perciben en ellos mismos una mejor capacitación que los docentes de Costa Rica $(36 \%)$. Actualmente la formación docente no prepara al educador para abordar las necesidades individuales de sus estudiantes.

Un alto porcentaje de docentes de Costa Rica y Chile, cree que la educación inclusiva es posible. Se observa que esta opinión es similar en ambos colectivos; sin embargo, en el caso de los docentes chilenos esta posición es más decidida (76\%) (Sanhueza, et al.,2012), esta postura coincide con los resultados de la presente investigación, donde todos los docentes encuestados están comprometidos con la educación inclusiva pues cumplen con la dimensión afectiva, respecto a los indicadores, el $98.1 \%$ da a apoyo académico de acuerdo a la necesidad especial de sus alumnos; el 97.2\% proporciona tiempo adicional a la clase y el $99.1 \%$ motiva a sus estudiantes antes de iniciar la clase
(Tabla 3).

En un estudio comparativo entre docentes de Costa Rica y Chile, los docentes manifiestan que no cuenta con las condiciones adecuadas para generar practicas pedagógicas mas inclusivas, Asimismo, un alto porcentaje de los docentes aplica adecuaciones curriculares significativas $72 \%$ (Sanhueza, et al.,2012), en nuestra investigación el $100 \%$ de los encuestados cumplen con los indicadores de la dimensión conductual, adecuando la sesión de enseñanza-aprendizaje a la necesidad especial de sus alumnos, el $94.3 \%$ prepara o prepararía material educativo adicional, el $100 \%$ adapta o adaptaría la sesión de aprendizaje, el 93.4\% aplica o aplicaría una evaluación diferenciada y el $99.1 \%$ acompaña o acompañaría académicamente a sus estudiantes (Tabla 4).

En una investigación realizada sobre actitud del docente hacia la educación inclusiva y los estudiantes con necesidades educativas especiales (Sevilla, et al., 2018) se encontró que la actitud de los docentes es negativa a diferencia de nuestro estudio donde se evidencia una actitud positiva (Tabla 3 y 4) para realizar adecuaciones a favor de los estudiantes con NEE. Sevilla sostiene que la actitud negativa hacia la educación inclusiva se volvía más positiva al referirse a los estudiantes con necesidades educativas especiales, adicionalmente se encontró que la edad, el sexo, el área de formación inicial y el nivel educativo en que labora el docente son factores que influyen en su actitud.

\section{CONCLUSIONES}

1. La actitud de los docentes de la Universidad Nacional Toribio Rodríguez de Mendoza de Amazonas frente a los estudiantes con necesidades educativas es positiva por que cumplen con las dimensiones: afectiva y conductual.

2. El $100 \%$ de docentes no cumple con los indicadores de la dimensión cognitiva, no presentan: Grado académico, especialización, diplomados, cursos, tampoco capacitaciones en TICs para educación inclusiva.

3. Los docentes cumplen con los indicadores de la dimensión afectiva: Apoyo académico (98.1\%), proporciona tiempo adicional (97.2\%), motivación antes de iniciar la clase (99.1\%). 
4. Los docentes cumplen con los indicadores de la dimensión conductual: Prepara o prepararía material educativo adicional $(94.3 \%)$, adapta o adaptaría la sesión de aprendizaje (100\%), aplica o aplicaría una evaluación diferenciada (93.4\%), acompaña o acompañaría académicamente a sus estudiantes $(99.1 \%)$.

\section{REFERENCIAS BIBLIOGRÁFICAS}

Aguado, M. T.; Gil, I.; Mata, P. El Enfoque intercultural en la formacion del profesorado: Dilemas y propuestas. Revista Complutense de Educación, Madrid, vol. 2, $\mathrm{N}^{\circ} 19$, p. 275-292, 2008.

Baron, R. y Byrne, D. (2002). Psicología social. 8 ed. Madrid: Prentice-Hall.

Castillo, A. Actitudes del profesorado de conservatorios sobre la integración educativa: un análisis exploratorio. Revista Española de Orientación y Pedagogía, 18(1): 47-58, 2007.

Díaz, F.; Hernández, G. Estrategias docentes para un aprendizaje significativo. México: McGrawHill, 2002.

Echeita, G.; Verdugo, M. La Declaración de Salamanca sobre necesidades educativas especiales diez años después: valoración y prospectiva. Salamanca: INICO, 2004.

Essomba, M. A. Liderar escuelas interculturales e inclusivas: equipos directivos y profesorado ante la diversidad cultural y la inmigración. Barcelona: Grao, 2006.

Gonzales, Y.; Triana, D., Actitudes de los docentes frente a la inclusión de estudiantes con necesidades educativas especiales. Revista Educación y educandos 21 (2):200-218, 2018.

Ministerio de Educación Chile. Decreto $\mathrm{N}^{\circ} 1$. Instructivo sobre proyectos de integración escolar. Santiago de Chile, 1998. Decreto $\mathrm{N}^{\circ}$ 170. Fija normas para determinar los alumnos con necesidades educativas

Mula, A. et al. (2002). Incidencias de las actitudes y expectativas de alumnos y profesores sobre el desarrollo del programa de integración. Universidad de Alicante.

Sanhueza, S.; Granada, M.; Bravo, L. Actitudes del profesorado de Chile y Costo Rica hacia la inclusión educativa. Cadernos de pesquisa, Brasil, Vol.42 N¹47, p.884-899, 2012.
Sevilla, D; Martí, M; Jenaro, C. Actitud del docente hacia la educación inclusiva y hacia los estudiantes con necesidades educativas especiales. Revista Innovación Educativa, Vol. $18 \mathrm{~N}^{\circ}$ 78, p.115-142, 2018

Terán, M.; Pachano, L. El Trabajo cooperativo en la búsqueda de aprendizajes significativos en clase de matemáticas de la educación básica. Revista Educare, Vol. $44 \mathrm{~N}^{\circ} 13$, p. 159-167, 2009.

Zerpa, C. Aprendizaje cooperativo en estrategias de comprensión de la lectura. Revista Pedagogía, Caracas, Vol. $67 \mathrm{~N}^{\circ} 23$, p. 187224, 2002. 